\title{
Statistical Analysis of Accuracy in Noise Figure Measurements
}

\author{
N. Otegi, J.M. Collantes, M. Sayed ${ }^{1}$ \\ Electricity and Electronics Department, University of the Basque Country, Apdo. 644, 48080 Bilbao, Spain \\ Phone: +34-94-601-2464, Fax: +34-94-601-3071, Email: jmcollan@we.lc.ehu.es \\ ${ }^{1}$ Microwave \& MillimeterWave Solutions, Santa Rosa, California, USA
}

\begin{abstract}
There is a growing trend to integrate noise figure measurement capabilities in microwave instruments originally devoted to other general purpose measurements as Vector Network Analyzers or Spectrum Analyzers. This opens up new possibilities to take into account vector corrections (at least in the case of VNA), or even to consider other techniques (such as cold-source) for computing the noise figure, different from the classical Y-factor approach used by most noise figure meters. In order to evaluate the suitability of these new options it is important to correctly analyze the benefits in terms of final accuracy that they can provide. However, errors in noise figure measurements have very different sources and nature, which sometimes do not allow straight forward conclusions on measurement accuracy. In this paper, a consistent evaluation of systematic errors and measurement uncertainty associated to different methodologies is presented. For that, the recommendations of the IEC-ISO Guide to the Expression of Uncertainty in Measurement (GUM) are followed. Some representative examples are given to illustrate the interest of the analysis.
\end{abstract}

Keywords - noise figure measurements, microwave circuits, uncertainty, systematic error.

\section{INTRODUCTION}

Significant efforts are being made in order to develop instruments that integrate different types of measurements in a unique system, in an attempt to optimize the time and equipment required for a complete device characterization. In particular, the inclusion of noise figure measurement capabilities in diverse microwave instrumentation, such as Vector Network Analyzers (VNA) or Spectrum Analyzers (SA), gives to noise figure measurements new possibilities further than the desired resource simplification. With new measurement capabilities the basic formulations ( $Y$-factor, cold-source) can be complemented with additional correction terms in order to eliminate errors arising from systematic effects, such as mismatch related effects, noise added by the receiver, etc.

Nevertheless, the final accuracy of a noise figure measurement depends on very diverse factors such as the characteristics of the device under test (DUT) and measurement setup, the methodology that is used for noise figure calculation, the hardware architecture of the measurement device etc. Different measurement approaches can lead to drastically different results for a given DUT, since the suitability of a particular methodology, having a specific level of corrections, is a function of the DUT and setup characteristics. In some cases, complicating the measurement process to include correction terms will not necessarily lead to more accurate results, while in other cases corrections may be crucial for achieving the desired accuracy. In addition, there is also error arising from random effects (such us connector variability, jitter...), uncertainty related with the limited accuracy of the measurement instruments; with the imperfect knowledge of the hot and cold noise temperatures, etc.

Therefore, at the moment of evaluating these new and more performing equipments for noise figure characterization, some questions arise concerning the basic strategy and formulation to be used, the hardware characteristics of the setup, the real benefits of each systematic error correction, the acceptable compromise between accuracy and measurement complexity, etc.

Traditional accuracy analyses associated to noise figure measurements have been limited to uncertainty evaluations of very particular measurement methodologies (scalar $Y$ factor) [1]. In this paper, a rigorous evaluation of systematic errors and measurement uncertainty of different noise figure characterization methodologies is presented. The recommendations of the IEC-ISO Guide to the Expression of Uncertainty in Measurement (GUM) [2] are followed in this analysis. In particular, GUM recommendation on how to deal with systematic and random effects is especially helpful in order to correctly identify the influence of systematic errors and measurement uncertainty. As was presented in [3], an independent formulation of the overall systematic error and the uncertainty allows a realistic comparison between the different characterization strategies.

Some basic concepts about uncertainty estimation according to GUM and the approach followed in the analysis are summarized in the following section. Details on the modeling of the noise figure measurement setup and the basic calculation methodologies are given in section 3 . Finally, application examples are given in section 4 in order to illustrate the benefits of a rigorous accuracy analysis.

\section{FUNDAMENTALS ON SYSTEMATIC ERROR AND UNCERTAINTY ESTIMATION}

GUM provides general rules for evaluating and expressing uncertainty associated with the conceptual 
design and theoretical analysis of methods and experiments in complex measurement systems. Thus, the analysis relies on basic concepts and notions from GUM. Some of the most important fundamentals utilized in this work are summarized in this section.

Measurement imperfections of diverse origin give rise to errors in the measurement result. Thus, in general, the result of a measurement is only an approximation or estimate of the value of the measurand. If the error is originated from a systematic effect it is called systematic error. Systematic error is defined in [4] as the mean that would result from an infinite number of measurements of the same measurand carried out under repeatability conditions minus a true value of the measurand. It is assumed in [2] that the result of a measurement has been corrected for all recognized significant systematic effects and that every effort has been made to identify such effects. If its value were insignificant to measurement uncertainty, a correction for a systematic effect could be ignored.

According to [2], uncertainty is formally defined as a parameter associated with the result of a measurement that characterizes the dispersion of the values that could be reasonably attributed to the measurand. Error and uncertainty represent completely different concepts. Indeed, the uncertainty of a measurement result reflects the lack of exact knowledge of the value of the measurand. The result of a measurement after correction for recognized systematic effects is still only an estimate of the value of the measurand because of the uncertainty arising from random effects and from imperfect knowledge of the correction factors for systematic effects.

The uncertainty is called standard uncertainty when it is expressed as a standard deviation (combined standard uncertainty if the result of a measurement is obtained from the values of other quantities). If all the quantities on which the result of the measurement depends are varied, the combined standard uncertainty can be evaluated by statistical methods. Let us consider a measurand $Y$ that is determined from other quantities $X_{1}, X_{2}, \ldots, X_{n}$ through a function $f$, where $f$ includes all corrections and correction factors that are taken into account.

$$
Y=f\left(X_{1}, X_{2}, \ldots, X_{n}\right)
$$

An estimate of the measurand $y$ can be obtained from the arithmetical mean of a set of $m$ independent observations of $Y$ :

$$
y=\bar{Y}=\frac{1}{m} \sum_{k=1}^{m} Y_{k}=\frac{1}{m} \sum_{k=1}^{m} f\left(X_{1, k}, X_{2, k}, \ldots, X_{n, k}\right)
$$

This way of averaging, rather than using the arithmetical means of the individual observations $y=f\left(\overline{X_{1}}, \overline{X_{2}}, \ldots \overline{X_{n}}\right)$, may be preferable when $f$ is a nonlinear function and correlation between variables need to be considered, as in the case of noise figure calculation.

The use of the term true value of the measurand is carefully avoided by [2] because it represents an unknowable quantity and, therefore, so does the error. Indeed, the measurement result is considered the best estimate of the value of the measurand and the result is qualified by a standard uncertainty, under the assumption that the result has been corrected for all recognized significant systematic effects. However, corrections for systematic effects may not always be possible, either because of an unacceptable increase in time, calibration complexity, required equipment, etc. In fact, some common noise figure measurement methodologies inherently ignore some systematic effects. Besides, valuable time could be lost in negligible corrections. Therefore, it is mandatory to identify the impact of the different systematic effects in the final measurement result.

In some cases, attempts are made to take the systematic effect into account by "enlarging” the uncertainty assigned to the result. As recommended by [2], this practice has been avoided in this work. Hence, in order to compare different noise figure strategies with different level of corrections, an estimation of the systematic error along with the measurement uncertainty is calculated using a Monte Carlo simulation. Specific guidance on obtaining measurement uncertainty by means of Monte Carlo process can be found in [5]. For the calculation, conventional true values and standard uncertainties are assigned to every parameter involved in it. Then, noise figure is calculated a large amount of times (in the standard manner of Monte Carlo methods) from parameter values that are randomly chosen from the probability distributions assigned to each parameter. From these results a mean value $m$, which is our estimate of the measurement result, and a standard deviation $\sigma$ are calculated. The standard deviation directly provides the combined standard uncertainty $u_{c}=\sigma$. The systematic error is estimated as: $e_{s}=m-F_{\text {true }}$, where $F_{\text {true }}$ is the noise figure obtained from the true values. This independent formulation of systematic errors and uncertainty provides a rigorous methodology to correctly choose an efficient measurement strategy for a given DUT and measurement receiver. This will be clearly evidenced by the application examples.

\section{BASIC NOISE FIGURE MEASUREMENT METHODOLOGIES AND CORRECTIONS FOR SYSTEMATIC EFFECTS}

Fig. 1 shows a generic noise figure measurement setup. This setup includes a noise source characterized by its two temperature states (cold and hot, $T_{c}, T_{h}$ ) having a corresponding reflection coefficient $\left(\Gamma_{s_{-} c}, \Gamma_{s_{\_} h}\right)$. The noise receiver is characterized by its input reflection coefficient $\left(\Gamma_{r e c}\right)$, its gain-bandwidth product $\left(B G_{r e c}\right)$ and its four noise 
parameters $\left(F_{\min }, R_{n}, \Gamma_{\text {opt }}\right)$. Finally, the DUT is defined by its $S$ parameters and by its four noise parameters $\left(F_{\text {min }}\right.$ ', $\left.R_{n}{ }^{\prime}, \Gamma_{\text {opt }}{ }^{\prime}\right)$.

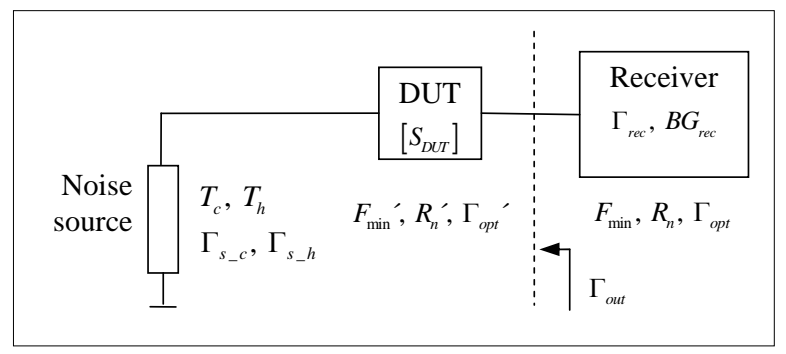

Fig. 1. Noise figure measurement setup

The two basic formulations for the calculation of noise figure are considered here: the $Y$-factor technique, used by the most common commercially available instruments for noise figure measurement, and the cold-source technique. The bases of these two techniques are widely reported in the literature [6]-[7]. Only a short summary is given here.

In the $Y$-factor technique, the noise figure of the DUT is computed from two noise powers $\left(N_{h}, N_{c}\right)$ measured with the noise source at its hot and cold temperatures $\left(T_{h}, T_{c}\right)$ respectively. Assuming that the cold temperature $T_{c}$ is equal to the reference temperature $T_{0}=290 \mathrm{~K}$, the noise figure can be obtained from (3), which constitutes the simplest formulation for noise figure calculation.

$$
F=\left(\frac{T_{h}}{T_{0}}-1\right) /\left(\frac{N_{h}}{N_{c}}-1\right)
$$

The cold-source technique computes the noise figure from a single noise measurement $\left(N_{c}\right)$ with a 50-Ohm source impedance, at room temperature, connected to the input of the DUT. For that, the gain-bandwidth product of the receiver $\left(B G_{r e c}\right)$ and the available gain of the DUT $\left(G_{a v}\right)$ have to be previously determined. Again, assuming that the room temperature is equal to the reference temperature $T_{0}$, the simplest formulation for cold-source is:

$$
F=\frac{N_{c}}{G_{a v} \cdot T_{0} K B G_{r e c}}
$$

The basic $Y$-factor and cold-source formulations represented by (3) and (4) can be complemented with the inclusion of correction terms to account for systematic effects, such as mismatch related effects, noise added by the receiver, etc. The most significant corrections that can be taken into account are listed here:

- De-embedding the DUT noise figure from the whole system noise figure (second stage correction).

- Calculation of the DUT available gain required for a rigorous application of the previous de-embedding. Classical Y-factor based approaches compute the DUT insertion gain from scalar power measurements. Both gains are similar for perfect match but they can diverge significantly when DUT output match degrades.

- Vector corrections accounting for any access network that may be placed at the input and output ports of the DUT (on-wafer probes, adapters, etc...).

- Differences between the cold temperature $\left(T_{c}\right)$ and the reference temperature $\left(T_{0}=290 \mathrm{~K}\right)$.

- Variations in the reflection coefficient of the noise source from cold to hot temperatures $\left(\Gamma_{s_{c}} \neq \Gamma_{s_{h} h}\right)$. Obviously, this effect cannot be completely removed from the $Y$-factor formulation, since that would require the knowledge of the DUT noise parameters, which are $a$ priori unknown.

- Dependence of the receiver noise figure on the source termination through the set of four noise parameters $\left(F_{\text {min }}\right.$, $\left.R_{n}, \Gamma_{\text {opt }}\right)$.

\section{APPLICATION EXAMPLES}

In this section, several noise figure measurement methodologies are analyzed. These methodologies include the most basic techniques, which can be implemented in a common noise figure meter or in a SA, or complex implementations that require vector measurements in a VNA. The aim of the work being to compare the suitability of different measurement approaches, equal noise generation properties are considered, at first, for the receivers; i.e., equal match and noise parameters are selected for each methodology.

The considered DUT is an amplifier with the following characteristics: $S_{21}=10 \mathrm{~dB} \angle 7.9^{\circ}, S_{11}=0.48 \angle 102^{\circ}, S_{22}=$ $0.41 \angle 43^{\circ}$ and $F \approx 3 \mathrm{~dB}$. A commercially available waveguide noise source, with Excess Noise Ratio $($ ENR $)=10.1$ $\mathrm{dB}$ is utilized. The reflection coefficients at the measurement frequency for both cold and hot temperatures are: $\Gamma_{s_{-} c}=0.15 \angle-20^{\circ}$ and $\Gamma_{s_{-} h}=0.08 \angle 24^{\circ}$. In fact, the actual noise figure of the DUT for $\Gamma_{s{ }_{c}}=0.15 \angle-20^{\circ}$ will be considered as the reference value, $F_{\text {true }}$, in the analyses. The receiver presents the following parameters: $F_{\min }=6.7$ $\mathrm{dB}, R_{n}=60 \Omega \Gamma_{\text {opt }}=0.08 \angle 271^{\circ}, \Gamma_{\text {rec }}=0.085 \angle 223^{\circ}$. Typical standard uncertainties, given by manufacturer's specifications, are assumed for the Excess Noise Ratio (ENR), for every variable associated to scattering measurements, for the receiver noise parameters and for the noise power measurements.

\section{A. Basic and Fully Corrected Methodologies}

The most basic methodology for noise figure measurement is considered first. This is a standard $Y$-factor technique in which only scalar power measurements are used $\left(Y F_{1}\right)$. The only systematic effect taken into account is the de-embedding of the second stage noise. $Y F_{1}$ is a widely extended approach used by typical noise figure meters. The obtained systematic error and combined 
standard uncertainty are plotted versus DUT input reflection coefficient in Fig. 2. While the uncertainty remains constant with DUT input match, an increase of the systematic error as the input match worsens can be observed. In contrast, an implementation of the $Y$-factor technique in a VNA $\left(Y_{2}\right)$ can be considered, with full correction for the different systematic effects: calculation of DUT available gain from $S$-parameter measurements, dependence of the receiver noise on the source termination and correction for the difference of the reflection coefficient of the noise source between its hot and cold states $\left(\Gamma_{s_{-} c} \neq \Gamma_{s_{-} h}\right)$. Results of the analysis for the $Y F_{2}$ methodology are plotted in Fig. 3. As it can be seen, systematic error is eliminated, while an increase of the uncertainty due to uncertainty of the correction terms can be signaled.

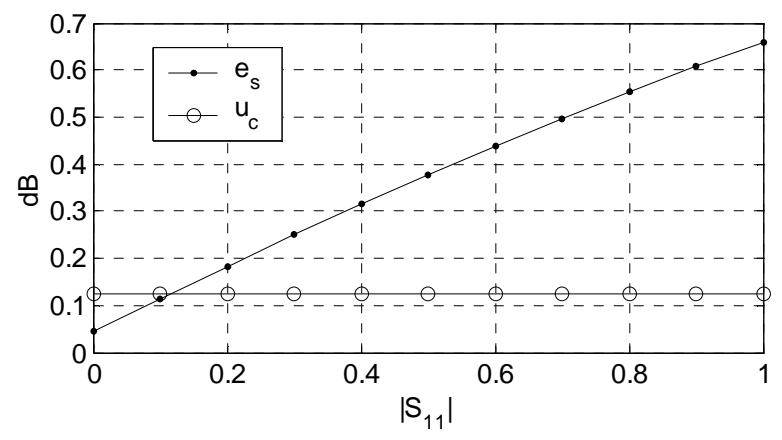

Fig. 2.Y-factor based methodology $Y F_{1}$. Only correction for second stage noise is taken into account.

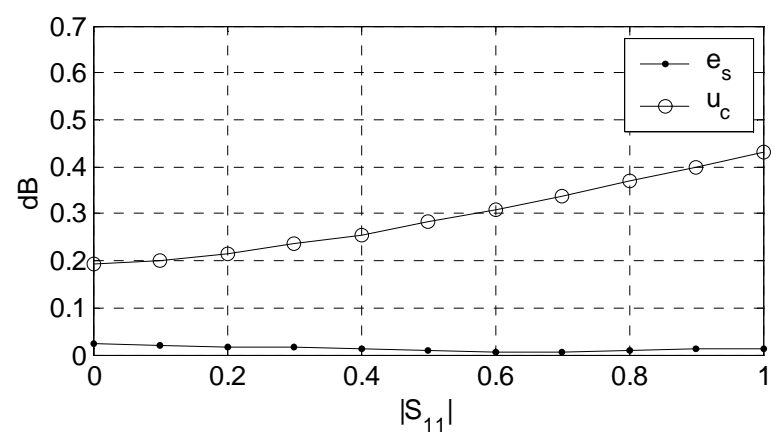

Fig. 3. $Y$-factor based methodology $Y F_{2}$. Corrections account for second stage noise, DUT available gain, receiver noise figure variation with source termination and changes in the reflection coefficient of the noise source $\left(\Gamma s \_c \neq \Gamma s \_h\right)$.

Let us consider a cold-source based methodology $\left(C S_{1}\right)$ obtained exclusively from scalar measurements. This could be implemented by means of a SA with noise measurement capabilities. In this case, the gain of the DUT can be obtained by means of a signal generator. This gain can be used in the cold-source formulation (equation (4)) instead of the available gain of the device. By nature, cold-source methodologies minimize the effects of noise source reflection coefficient variations from hot to cold state since, during DUT measurement, the noise source only operates at its cold state. However, in a similar manner than in the scalar $Y$-factor technique, mismatch effects can affect this methodology. In this case, differences between the reflection coefficients of the noise source in its cold state and the generator used for the insertion gain calculation are crucial as the input match of the DUT worsens. For this $C S_{1}$ analysis, a generator with a source match of $28 \mathrm{~dB}$ is considered. Results plotted in Fig. 4 show a considerably lower systematic error than the scalar $Y$-factor. Besides, measurement uncertainty, which remains constant except for perfect match, is noticeably higher than in the $Y F_{1}$ case.

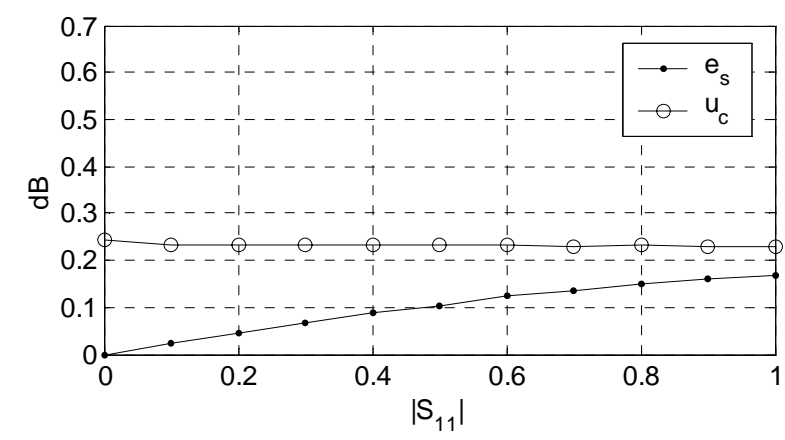

Fig. 4. cold-source based scalar methodology $\mathrm{CS}_{1}$. Only correction for second stage noise is taken into account and in addition DUT available gain is substituted by its insertion gain.

As in the $Y$-factor case, a fully corrected cold-source methodology $C S_{2}$ is also considered. This methodology could be implemented in a VNA. $C S_{2}$ adds to the basic formulation (4) all the available correction terms. Results show in Fig. 5 a negligible systematic error for all the input match range. This result can be compared with the one obtained in Fig. 3 for $Y$-factor based methodology $Y F_{2}$, which has a comparable level of correction terms. It can be observed that $C S_{2}$ presents higher uncertainty for low input reflection coefficient of the DUT, while it is lower for worse input match.

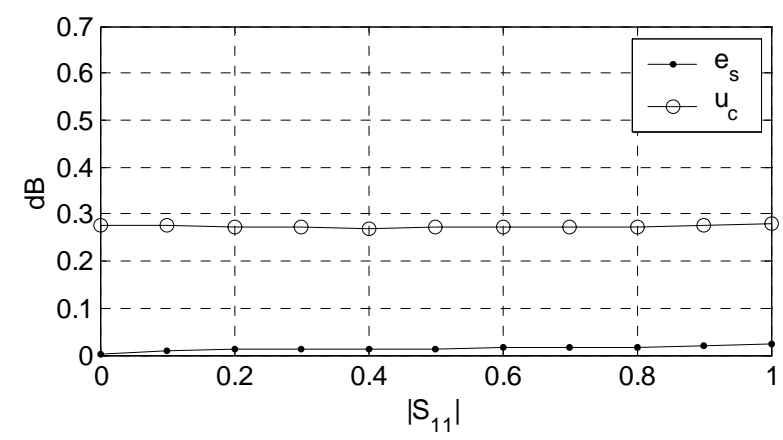

Fig. 5. cold-source based scalar methodology $\mathrm{CS}_{2}$. Corrections account for second stage noise, DUT available gain and receiver noise figure variation with source termination. 


\section{B. Evaluation of Corrections for Systematic Effects}

The previous results have evidenced that for this DUT approaches limited to scalar measurements $\left(Y F_{1}, C S_{1}\right)$ can be critically affected by mismatch effects, which have been eliminated in the fully corrected $Y F_{2}$ and $C S_{2}$ methodologies. However, these methodologies include non-trivial calibrations (noise calibration of the receiver for example) that complicate and slow down the whole measurement process. As the contribution of the receiver noise is attenuated by the device gain, the influence of such a noise calibration can be analyzed as a function of the device gain. For that, the systematic errors obtained with three different methodologies, two $Y$-factor based $\left(Y F_{3}, Y F_{4}\right)$ and a cold-source one $\left(C_{3}\right)$, are calculated as a function of DUT gain $\left(S_{21}\right) . Y F_{3}$ adds to the scalar approach $Y F_{1}$ the calculation of the available gain of the device, while $Y F_{4}$ adds also a correction for the changes in the reflection coefficient of the noise source. Therefore, the only difference between $Y F_{4}$ and the fully corrected $Y F_{2}$ is the lack of noise calibration of the receiver in the former. $\mathrm{CS}_{3}$ is the standard cold-source technique given by equation (4) with a second-stage correction and without noise calibration of the receiver. The obtained results are plotted in Fig. 6. As it can be observed, both $Y F_{4}$ and $C S_{3}$ eliminate the systematic error for high DUT gain and therefore, noise calibration of the receiver could be neglected in that case. On the contrary, the lack of noise calibration of the receiver can drastically degrade the accuracy of the result for low gain devices, even if vector corrections are applied [8]. It should be noted that the $Y$ factor technique requires the knowledge of the reflection coefficient of the noise source in its hot state for properly eliminating mismatch effects, as evidences the nonnegligible systematic error obtained in the $Y F_{3}$ approach, even for high DUT gain.

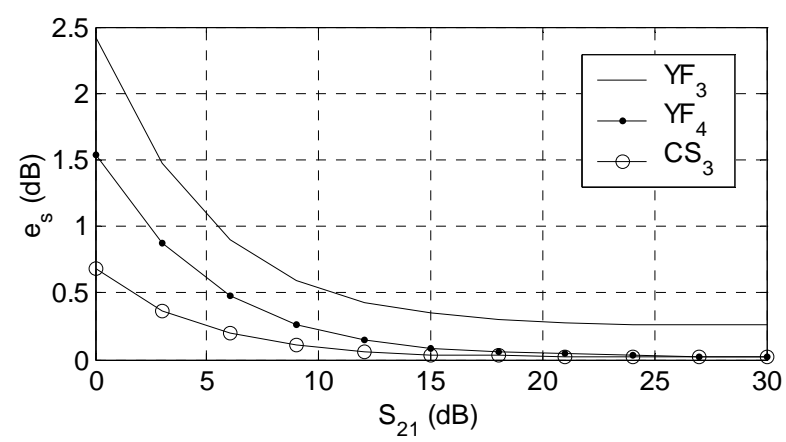

Fig. 6. comparison of the systematic error obtained with three different methodologies: $Y$-factor based $\mathrm{YF}_{3}$ and $Y F_{4}$, and coldsource based $\mathrm{CS}_{3}$. Corrections in $Y F_{3}$ account for second stage noise and DUT available gain, while $Y F_{4}$ adds also a correction for $\left(\Gamma S \_c \neq \Gamma s \_h\right) . C S_{3}$ is the standard cold-source methodology.

\section{Systematic effects from Hardware Characteristics of Noise Receivers}

The analyses above have focused on different measurement methodologies, with different correction levels, that could be performed in nowadays available noise receivers. No particular characteristics of the instruments have been taken into account. Nevertheless, these receivers have architectural differences that actually affect the noise figure measurement accuracy. One important hardware characteristic of a receiver is that of performing single side band (SSB) measurements, as is the case of a commercial noise figure meter or a SA, or double side band (DSB) measurements, as in the case of a VNA. In the SSB measurement case, only noise contribution from the interest frequency is measured. On the contrary, in the DSB case, noise from the image frequency, as well as noise converted by the odd harmonics of the local oscillator (LO) of the receiver is added to the interest noise. Generally, noise contribution from the image is not critical if sufficiently low intermediate frequency (IF) is chosen in the measurement (unless abrupt changes in the device characteristics occur). However, harmonic noise conversion from undesired frequencies can still seriously degrade the measurement performed in a VNA.

The impact of the harmonic noise conversion in the final result is obviously influenced by the characteristics of the DUT at the harmonic conversion frequency. As an example, two different measurement methodologies (a standard $Y$-factor technique, $Y F_{D S B}$, and a standard coldsource, $C S_{D S B}$ ) implemented in a VNA where harmonic noise conversion is not prevented are considered. The systematic error obtained for these methodologies is analyzed as a function of DUT gain at the measurement frequency $\left(S_{21}\right.$ parameter) for several harmonic noise conversion contributions (as a percentage of the noise power at the measurement frequency). It is important to remark that the contribution of harmonic noise conversion varies from calibration stage, where a broadband noise source is connected to the receiver, to the measurement one, where this contribution is also defined by the characteristics of the DUT (gain, noise figure and match) at the harmonic conversion frequency. For these examples, a $10 \%$ of spurious noise is added in the hot and cold noise powers measured in the calibration of the receiver, while five values from $2 \%$ to $10 \%$ are chosen for the measurement process.

In order to eliminate the influence of other systematic effects, perfect match is considered for both noise source and DUT.

The systematic errors obtained for the $Y F_{D S B}$ analysis are shown in Fig. 7. As it can be seen, the spurious noise contributions do not affect the results for high DUT gain. In contrast, for low gain of the device, the systematic error increases as the noise contribution in measurement diverges from the value of calibration. Results obtained for $C S_{D S B}$ are given in Fig. 8. It can be observed that 
systematic errors decrease with device gain increase, but do not tend to cero, as in the $Y F_{D S B}$ case. Again, systematic error augments as the difference in the spurious noise contribution from calibration to measurement increases.

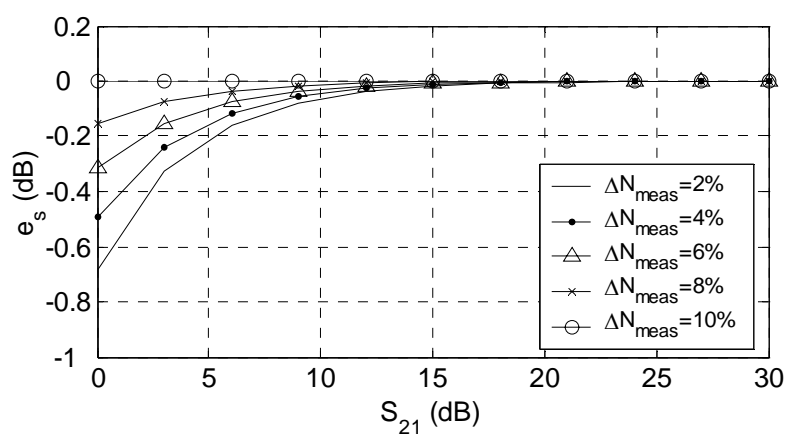

Fig. 7. standard $Y$-factor methodology $Y F_{D S B}$ from $D S B$ measurements.

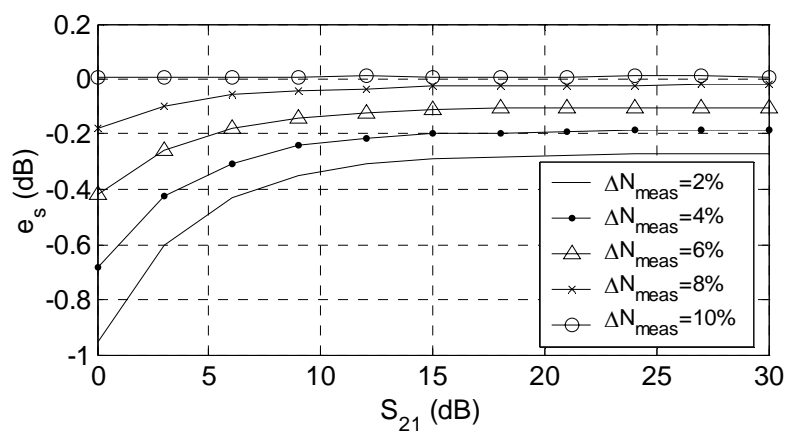

Fig. 8. standard cold-source methodology $C S_{D S B}$ from DSB measurements.

As it can be concluded from these examples, harmonic noise conversion can significantly affect the final accuracy of noise figure measurements. Besides, it should be reminded that a common noise figure meter or a SA are not affected by harmonic conversion, due to their architecture.

Finally, it is important to remark that most of the results discussed in these examples rely on the independent handling of systematic error and uncertainty. Actually combining both in a single quantity, as in [9], can result in a loss of valuable information at the time of making decisions about the methodology to use, the design of the setup or the importance of a particular systematic effect for achieving a required accuracy, etc.

\section{CONCLUSION}

A rigorous statistical analysis of accuracy in noise figure measurements has been presented. The independent treatment of systematic errors and uncertainty allows a comparative analysis of different methodologies with different level of corrections that could be implemented in different noise receivers. Reliable information can be extracted about the suitable strategy and formulation to be used, the hardware characteristics of the setup, the real benefits of each systematic error correction or even the required balance between measurement accuracy and measurement complexity. Examples carried out on an amplifier are given to illustrate the benefits of a rigorous accuracy analysis.

\section{ACKNOWLEDGMENT}

The authors wish to thank the University of the Basque Country for partially funding this work.

\section{REFERENCES}

[1] D. Boyd, "Calculate the Uncertainty of NF Measurements," Microwaves \& RF, October 1999, pp. 93-102

[2] ISO-IEC-OIML-BIPM, "Guide to the Expression of Uncertainty in Measurement,” 1993, corrected 1995.

[3] N. Otegi, J. M. Collantes, M. Sayed, "Uncertainty Estimation in Noise Figure Measurements at Microwave Frequencies", IEEE International Workshop on Advanced Methods for Uncertainty Estimation in Measurements, Niagara Falls, Ontario, Canada, pp. 84-89.

[4] "International Vocabulary of Basic and General Terms in Metrology", $2^{\text {nd }}$ Edition, 1993.

[5] ISO-IEC-OIML-BIPM, "Guide to the Expression of Uncertainty in Measurement. Suplement 1. Numerical methods for the propagation of distributions," 2004.

[6] "Fundamentals of RF and Microwave Noise Figure measurements," Agilent Application Note 57-1

[7] R. Meierer, C. Tsironis, "An On-Wafer Noise Parameter Measurement Technique with Automatic Receiver Calibration, ", Microwave Journal, March 1995, pp. 22-37.

[8] J.M. Collantes, R.D. Pollard, M. Sayed, "Effects of DUT Mismatch on the Noise Figure Characterization: A Comparative Analysis of Two Y-Factor Techniques," IEEE Transactions on Instrumentation and Measurement, Vol. 51, no. 6, December 2002, pp. 1150-1156.

[9] A. Collado, J. M. Collantes, L. De la Fuente, N. Otegi, L. Perea, M. Sayed, "Combined Analysis of Systematic and Random Uncertainties for Different Noise-Figure Characterization Methodologies," IEEE MTT-S International Microwave Theory and Techniques Symposium, Philadelphia, PA, June 2003 pp. 14191422 